\title{
Research Article \\ Resonant Scattering and Energy Relaxation in Quantum Dot Systems
}

\author{
I. A. Larkin ${ }^{1}$ and A. Vagov ${ }^{2}$ \\ ${ }^{1}$ Institute of Microelectronics Technology Russian Academy of Sciences, Chernogolovka 142432, Russia \\ ${ }^{2}$ Institut für Theoretische Physik III, Bayreuth Universität, 95440 Bayreuth, Germany \\ Correspondence should be addressed to I. A. Larkin; vaniala2000@yahoo.co.uk
}

Received 26 February 2017; Accepted 16 April 2017; Published 11 May 2017

Academic Editor: Sergio E. Ulloa

Copyright ( 2017 I. A. Larkin and A. Vagov. This is an open access article distributed under the Creative Commons Attribution License, which permits unrestricted use, distribution, and reproduction in any medium, provided the original work is properly cited.

\begin{abstract}
We propose a new Auger-like mechanism for energy relaxation in quantum dots (QD) driven by resonant scattering of delocalized wetting layer (WL) carriers. It is demonstrated that resonant scattering leads to a considerable increase in the relaxation rate that can explain experimentally obtained relaxation rates. Analytical results for the relaxation rate are obtained for rectangular dots revealing a weak logarithmic dependence on the dot depth and level density. Comparing results for a rectangular and a parabolic QD model we conclude that the relaxation rate is not very sensitive to a chosen model.
\end{abstract}

\section{Introduction}

Capturing and subsequent relaxation of hot carriers (quasielectrons and holes) in nanoscale QD are of central importance for practical applications of QD systems, for example, in lasers [1] single photon emitters [2] and photodetectors with unique properties $[3,4]$. The discussion of the corresponding rate is controversial. It is long known that a singular spectrum in QD inhibits incoherent emission of phonons suppressing the most effective relaxation mechanism [5], so the term phonon bottleneck was coined. Indeed, experiments $[6,7]$ demonstrate a rapid enhancement of the relaxation rate when interlevel spacing coincides with phonon energy. However, in many experiments the bottleneck did not reveal itself and the relaxation rate was comparable or even faster than provided by phonons in bulk systems [8-15] possibly indicating the role of the continuous spectrum [16-19]. Further studies lead to a conclusion that the processes of capturing and relaxation can be separated and that the relaxation is likely to be defined by different mechanisms in specific situations for both capture and relaxation [13, 18, 20, 21].

Several relaxation scenarios were proposed to explain these experimental results: phonon-assisted tunnelling to impurity states [22], decay of the LO phonons [23], and several Auger-like mechanisms which included particle collisions [24], QD coupling with the WL plasma excitations [25], phonon-assisted intradot Auger interactions [26], and Auger interactions between excitons in the dot and in a localized state of the wetting layer [27]. Experimentally observed dependence of the relaxation rate on the number of created excitons prompted the conclusion that Auger-like processes are likely to play a role when exciton density is $n>10^{9} \mathrm{~cm}^{-2}$, while other mechanisms are dominant at smaller density $[13,20,28]$. Calculations of the rate due to WL carrier collisions [24, 29, 30] and plasma excitations [25] demonstrated that these mechanisms become important at $n>10^{11} \mathrm{~cm}^{-2}$ leaving the phonon-assisted intradot Auger interaction mechanism [26] as the likely candidate to explain the experimentally observed relaxation at lower densities [13].

However, as we show below, the Coulomb interaction between delocalized WL and trapped carriers can provide an efficient relaxation mechanism yielding a high relaxation rate even for as low as $n=10^{9} \mathrm{~cm}^{-2}$ density when the resonant scattering of the WL carriers is considered. This mechanism is general and could be applied to describe relaxation to deep states in a general gaseous system. Here we apply it to the QD systems. In the discussion we calculate the rate for two models of the confining potential: a rectangular model for which 
analytical results are obtained and a parabolic potential for which we do numerical simulations. Qualitative agreement between the two models is demonstrated, showing the results are not very sensitive to details of the chosen model. By substituting standard parameters for the InGaAs dot systems we obtain relaxation rate which agrees with experiments.

\section{Relaxation Rate}

Scattering of delocalized WL carriers induces transitions of the trapped carrier between the QD states leading to relaxation in the system the rate of which is obtained from the scattering cross-section as

$$
\frac{d \varepsilon_{\alpha}}{d t}=n \sum_{\beta} \int v_{2}\left(\varepsilon_{\beta}-\varepsilon_{\alpha}\right) \sigma_{\alpha \beta}(\lambda) n_{T}(\lambda) d \lambda,
$$

where $\sigma_{\alpha \beta}(\lambda)$ is the scattering cross-section at energy $\lambda$ of the WL carrier, $\alpha, \beta$ are initial and final two particle scattered states, where the energy of the trapped carrier is $\varepsilon_{\alpha, \beta}$, respectively, $v_{2}$ is initial velocity of the WL carrier, $n_{T}$ is temperature distribution of the wetting layer carriers, for which we take Boltzmann distribution, and the summation is done over all possible scattering states $\beta$. The cross-section $\sigma_{\alpha \beta}(\lambda)$ includes electron-electron, hole-hole, and electronhole scattering as well as carriers exchange. Hereafter all calculations are done in dimensionless Bohr units for InAs material.

Conventional calculations of $\sigma_{\alpha \beta}(\lambda)$ within the Fermi rule approximation $[24,29,30]$ overlook the fact that the energy dependence of the cross-section exhibits sharp resonances when $E=\lambda+\varepsilon_{\alpha}$ is close to energy of two particle virtual states where two carriers are temporarily confined in a QD during a time defined by the inverse width of the resonance. A schematic illustration of the resonant scattering as opposed to the direct scattering described by the Fermi rule is illustrated in Figure 1.

High density of resonances for the scattering of WL carriers with energy in the temperature interval is the main reason why resonant scattering may dominate the relaxation. For weak Coulomb interaction this density is estimated as the density of two particle states obtained under the condition that transitions to the delocalized WL states is neglected. For example, if we assume that a QD has an equidistant (degenerate) single particle spectrum with the gap $\delta_{e}=$ $30 \mathrm{meV}$ for electrons and $\delta_{h}=15 \mathrm{meV}$ for holes and $U=$ $200 \mathrm{meV}$ depth, the number of respective single particle states is $N_{e}=20$ and $N_{h}=80$ while the total number of excitonic states is approximately $N_{e} N_{h}=1600$. The states in the energy interval $-2 U<E<-U$ are stable, whereas the states $-U<E<0$ are virtual if transitions to the WL states are taken into account. The number of such states gives the number of resonances in the cross-section. The degeneracy of the virtual states is lifted by the intradot Coulomb interaction and by deviations from the symmetric shape of the dot. While these factors have only a minor effect on the low lying stable states, the energy levels of the higher excited states especially of the virtual states become randomized and insensitive to details of the single particle spectrum. As a result, positions of the resonances can be described by an averaged density of virtual states obtained by assuming a quasi-continuous approximation for the single particle levels which is valid despite the point like single particle QD spectrum. For $E=$ $-U / 2$, that is, when a trapped carrier has energy $\varepsilon \approx-U / 2$, the mean distance between the resonances is estimated as $2 U / N_{e} N_{h}=0.25 \mathrm{meV} \approx 3 \mathrm{~K}$ and, therefore, at $T \gg 3 \mathrm{~K}$ many resonances contribute in (1) enhancing the relaxation.

In the vicinity of a resonance $j$, the cross-section is given by the 2D Breit-Wigner expression [31]

$$
\sigma_{\alpha \beta}^{j}(\lambda)=\frac{4}{m_{2} v_{2}} \frac{\gamma_{j}^{\alpha} \gamma_{j}^{\beta}}{\left(\lambda+\varepsilon_{a}-E_{j}\right)^{2}+(1 / 4) \gamma_{j}^{2}},
$$

where $E_{j}$ is the energy of the resonance, $\gamma_{j}^{\alpha}$ is the partial width of the resonance, $\gamma_{j}=\sum_{\alpha} \gamma_{j}^{\alpha}$ is its total width, and $\lambda$ and $\varepsilon_{\alpha}$ are the energy of the scattered and the trapped carriers, respectively. Partial widths are found as

$$
\gamma_{j}^{\alpha}=2 \pi \sum_{k}\left|V_{j}^{\alpha}\right|^{2} \delta\left(E_{j}-\varepsilon_{\alpha}-\lambda\right),
$$

where $V_{j}^{\alpha}$ is the transition matrix element between the virtual state $j$ and a scattering state $\alpha$ and $E_{j}=\varepsilon_{j}+\lambda_{j}$ is the zeroth-order approximation for the resonance energy, where $\varepsilon_{j}$ and $\lambda_{j}$ are single particle QD levels for temporarily trapped carriers. Equation (1) is valid if neighboring resonances do not overlap; that is, $\gamma_{j}<E_{j}-E_{j+1}$. If the temperature is not too low $\gamma_{j} \ll T$ the summation over $\lambda$ in (1) for the crosssection (2) is carried out yielding for the rate

$$
\frac{d \varepsilon_{a}}{d t}=\frac{8 \pi n}{m_{2}} \sum_{j \beta} \frac{\gamma_{j}^{\alpha} \gamma_{j}^{\beta}}{\gamma_{j}}\left(\varepsilon_{\alpha}-\varepsilon_{\beta}\right) n_{T}\left(E_{j}-\varepsilon_{\alpha}\right),
$$

where the condition $\varepsilon_{\alpha}<E_{j}$ holds. In the quasi-continuous approximation (1) can be further simplified if the density of resonances does not change within the $\delta E=T$ interval. The number of contributing resonances in the sum in (1) can be approximated as $T / \bar{\Delta}$, where $\Delta$ is the average gap between neighboring resonances. This new $T$ factor cancels the normalization $T^{-1}$ in the Boltznmann distribution and the latter can be approximated by the delta function $n_{T}=$ $\delta(\lambda)$.

\section{Coulomb Interaction Matrix Elements}

For a rectangular shaped $l \times l$ QD single particle eigenfunctions are plane waves defined by a 2D wave vector $k$. Assigning indexes 1 and 2 to the trapped and free carrier, respectively, the zeroth-order two particle states are defined as pairs of single particle states. Coulomb transition matrix elements and the resonance widths are calculated with the following assumptions: (1) the confinement length in a direction perpendicular to the wetting layer is small and is disregarded in the calculations, which are strictly twodimensional, (2) the eigenstates are taken as $\exp (i k x)$, which is equivalent to periodic boundary conditions, and (3) the 


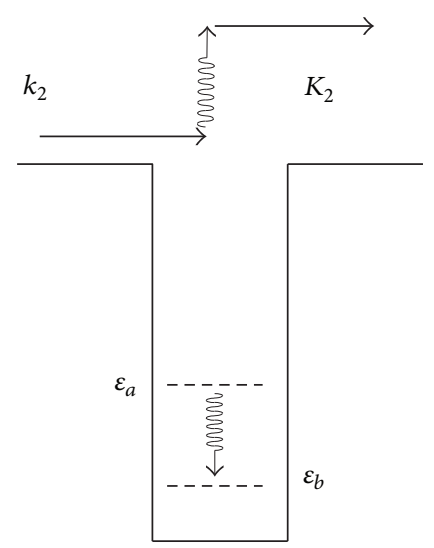

(a)

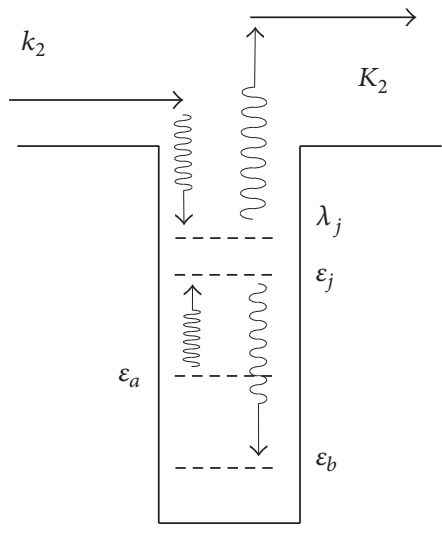

(b)

FIGURE 1: Schematic representation of direct (a) and resonant (b) scattering of a carrier with $k_{2}$ momentum on a dot with a trapped carrier on a level $\varepsilon_{\alpha}$. After collision the first carrier leaves carrying momentum $K_{2}$ and the second one remains trapped on a new level $\varepsilon_{\beta}$. Resonant scattering involves formation of a virtual level $j$.

Coulomb interaction is weak and acts only inside the dot, which allows us to expand the corresponding integrations over the entire space (with a proper normalization for the eigenfunctions). The latter assumption yields the already mentioned quasi-continuous approximation for the matrix elements which will be used in the sums over the discrete states in (1). With these assumptions (3) yields

$$
\gamma_{j}^{\alpha}(q)=\frac{(2 \pi)^{3}}{l^{4}} \frac{1}{q^{2}} \delta\left(\varepsilon_{j}+\lambda_{j}-\varepsilon_{\alpha}-\lambda\right)
$$

where $q=K_{1}-k_{1}=k_{2}-K_{2}$. It is convenient to introduce the quantity

$$
\begin{aligned}
\bar{\gamma}_{j}^{\alpha} & =\frac{l^{2}}{(2 \pi)^{2}} \int \gamma_{j}^{\alpha} \delta\left(\varepsilon_{j}-\varepsilon_{\alpha}-\Delta\right) d q \\
& =\frac{2 \pi}{l^{2}} \frac{1}{\left(\varepsilon_{\alpha}-\varepsilon_{j}\right)^{2}} \frac{v_{1}^{j} v_{2}^{j}|\sin (\Theta)|}{v_{1}^{j 2}+v_{2}^{j 2}-2 v_{1}^{j} v_{2}^{j} \cos (\Theta)}
\end{aligned}
$$

where $\Theta$ is angle between $k_{1}^{j}$ and $k_{2}^{j}$ and $v_{1(2)}^{j}=k_{1(2)}^{j} / m_{1(2)}$ is carrier velocity. Equation (6) defines the averaged width that corresponds to a particular energy change, $\Delta$, of the trapped carrier. In deriving (6) the momentum transfer was assumed small; $\left|k_{1}^{j}-k_{1}^{\alpha}\right| \ll k_{1(2)}^{j}$. As what follows from (6) transitions to neighboring states dominate the relaxation. Using (6) we write the rate (1), averaged over the degenerate initial and final states $\alpha$ and $\beta$, as

$$
\frac{d \varepsilon_{\alpha}}{d t}=\frac{16 \pi^{2} n}{m_{1} m_{2} l^{2}} \sum_{j, \varepsilon_{\beta}} \frac{\bar{\gamma}_{j}^{\alpha} \bar{\gamma}_{j}^{\beta}}{\gamma_{j}}\left(\varepsilon_{\beta}-\varepsilon_{\alpha}\right) \delta\left(\varepsilon_{j}+\lambda_{j}-\varepsilon_{\alpha}\right) .
$$

The summation over $\varepsilon_{\beta}$ and $j$ is done in the quasi-continuous approximation where sums are reduced to integrals. The limits of integration are obtained from the conditions $\varepsilon_{j}+\lambda_{j} \geq$ $\varepsilon_{\alpha(\beta)}$ and $\varepsilon_{\alpha(\beta)}<0$. The final expression for the rate reads

$$
\begin{aligned}
\frac{d \varepsilon_{\alpha}}{d t}= & -8 n \int_{\varepsilon_{\alpha}+\delta}^{0} \frac{d \varepsilon}{\varepsilon-\varepsilon_{a}}\left[\frac{\varepsilon+U}{\varepsilon_{\alpha}+U} \ln \left(\frac{U+\varepsilon}{\varepsilon-\varepsilon_{\alpha}}\right)-1\right] \\
\times \ln \left|\frac{v_{1}+v_{2}}{v_{1}-v_{2}}\right|, & \\
v_{1} & =\sqrt{2 \frac{U+\varepsilon}{m_{1}}}, v_{2}=\sqrt{2 \frac{U+\varepsilon_{a}-\varepsilon}{m_{2}}},
\end{aligned}
$$

where $\delta$ is cutoff equal to the QD single particle energy gap between $\varepsilon_{\alpha}$ and the next lower level. The logarithmic singularity at $v_{1}=v_{2}$ is an artifact of the approximation used in calculating the transition matrix elements; however, it disappears after integration. If a lighter WL electron scatters on a heavier trapped hole, the logarithmic singularity is far from $\varepsilon=\varepsilon_{\alpha}$ most contributing to the integral and reduces to a small factor $v_{1} / v_{2}<1$ in the resulting rate.

The integration in (8) can be easily done numerically; nevertheless, it is instructive to evaluate its largest part on the lower integration limit analytically which yields

$$
\begin{aligned}
& \frac{d \varepsilon_{\alpha}}{d t}=-8 n \ln \left|\frac{v_{1}+v_{2}}{v_{1}-v_{2}}\right|\left\{\frac{\pi^{2}}{6}+\ln \left[\frac{U \delta}{-\varepsilon_{\alpha}\left(U+\varepsilon_{\alpha}\right)}\right]\right. \\
& \left.\quad \times\left(\frac{1}{2} \ln \left[\frac{-\varepsilon_{\alpha} \delta}{U\left(U+\varepsilon_{\alpha}\right)}\right]+1\right)\right\},
\end{aligned}
$$

where $v_{1}=\sqrt{2\left(U+\varepsilon_{\alpha}\right) / m_{1}}$ and $v_{2}=\sqrt{2 U / m_{2}}$. In the limit of $U / \delta \gg 1$ and when the trapped carrier is still on the higher QD level, that is, $-\varepsilon_{\alpha} \ll U$, (9) yields

$$
\frac{d \varepsilon_{\alpha}}{d t} \propto-\ln \left(\frac{U}{\delta}\right)^{2}
$$

where $U / \delta$ is roughly equal to the total number of single particle QD states. One can see that the largest contribution to 
the relaxation rate depends logarithmically on the QD depth and the density of its single particle levels and is not sensitive to other details of the QD model. This gives an indication that the obtained result has an applicability range beyond the rectangular model QD.

To compare the resonant scattering contribution to that of the direct scattering (Figure 1(a)) we calculate the crosssection using the Fermi golden rule approximation. Substituting the result into (1) and using the same assumptions as before we obtain

$$
\frac{d \varepsilon_{a}}{d t}=-n \log \left|\frac{v_{1}+v_{2}}{v_{1}-v_{2}}\right| \log \left(\frac{U+\varepsilon_{a}}{\delta}\right) \propto \ln \left(\frac{U}{\delta}\right) .
$$

As (10), this expression also depends on the QD depth and level density logarithmically; however, here the logarithm is not squared. Consequently for deep dots with a large number of levels, $U / \delta \gg 1$, resonant scattering always dominates the relaxation.

Now we take a parabolic QD model where the confining potential is defined by $V(r)=-U+m_{1,2} \omega_{1,2}^{2} r^{2} / 2$ at $r<$ $a=\sqrt{2 U / m_{1,2} \omega_{1,2}^{2}}$ and $V=0$ otherwise. As above we assume that the interaction acts mainly inside a dot, which allows using harmonic oscillator eigenfunctions for both QD and WL states. The two particle states are defined by four quantum numbers $j=(n, m ; N, M)$ and $\alpha=(r, s ; R, S)$, where $n, N, r, R$ are the main quantum numbers and $m, M, s$, $S$ are the angular momenta of entering single particle states. The number $R$ which describes the scattered particle is given by the energy conservation condition $R=\left(U+\lambda_{1,2}\right) / \omega_{1,2}$ and can be, therefore, noninteger, leading to a divergency of the corresponding wave function at $r \rightarrow \infty$. However, in the calculation of the matrix elements this divergency is suppressed by the wave function with the main number $N$ that corresponds to a lower energy. The transition matrix element can be expressed as an integral

$$
\begin{aligned}
& V_{n m ; N M}^{r s ; R S} \\
& =(-i)^{|M-S|} i^{|m-s|} \delta(m+s-M-S) \\
& \quad \times \int_{0}^{\infty} d q \phi_{n+m}^{r-n+s-m}\left[\frac{q^{2}}{4 m_{1} \omega_{1}}\right] \phi_{n}^{r-n}\left[\frac{q^{2}}{4 m_{1} \omega_{1}}\right] \\
& \quad \times \phi_{N+M}^{R-N+S-M}\left[\frac{q^{2}}{4 m_{2} \omega_{2}}\right] \phi_{N}^{R-N}\left[\frac{q^{2}}{4 m_{2} \omega_{2}}\right],
\end{aligned}
$$

where $\phi_{n}^{m}(x)=\exp (x / 2) L_{n}^{m}(x) x^{m / 2}$ is the radial part of the oscillator wave function $\left(L_{n}^{m}(x)\right.$ denotes the Laguerre functions). Equation (12) is substituted into (3) and (1) that are evaluated numerically.

In the above analysis we assumed the scattering process at which the carriers are of a different type. For the carriers of the same type the cross-section is obtained by carrier permutation in the scattering amplitude $f(1,2)$ as $\sigma=|f(1,2)|^{2}+$ $|f(2,1)|^{2} \pm 2\left|f(1,2) f^{*}(2,1)\right|$. It follows from the calculations $|f(1,2)|^{2}$ and $|f(2,1)|^{2}$ provide an equal contribution to the rate, while the interference term $2\left|f(1,2) f^{*}(2,1)\right|$ leads to a much smaller contribution because it involves transitions with larger energy transfer. Consequently, the rate acquires an additional factor 2 . Another factor 2 comes from spin degeneracy of WL carriers. It must be noted that when carriers are of the same type the Coulomb blockade introduces an additional exponential factor $\exp \left(-E_{c} / T\right)$ into the rate. The value of $E_{c}$ for the InAs dots can be estimated as few meV and, therefore, the Coulomb blockade is noticeable at low temperatures.

\section{Results and Discussion}

Results of calculations for the relaxation rate are summarized in Figure 2. Direct comparison between considered models is not possible since they have a different structure of single particle levels. However, we utilize the fact that the level structure dependence in (9) and (10) comes only via the energy gap between neighboring states and that the rate is mainly defined by transitions to the nearest levels. It has to be noted that in the parabolic potential zeroth-order two particle states eigenvalues are degenerate, while in real dots this degeneracy is lifted as discussed above. To bypass this difficulty we formally assume a slightly different energy gap for the second particle $\delta_{1}=1.1 \delta_{2}$, which was enough to break the artificial degeneracy of the resonances imposed by the degeneracy of single particle QD states. We note that the quasi-continuous approximation is not needed for the parabolic model, where the summation in (1) is done numerically. The temperature dependence of the rate is weak as long as $\bar{\Delta} \ll T \ll \omega$, for the calculations we chose $T=40 \mathrm{~K}$.

The results in Figure 2 demonstrate that the relaxation due to resonant scattering is considerably faster for deep QD levels. One can also see that resonant scattering driven relaxation for both considered models is qualitatively similar, while quantitatively the models differ only by a factor 1.5. The rate increases in the interval $\varepsilon_{\alpha}<\delta$, then saturates, then abruptly drops, and becomes zero at $\varepsilon_{\alpha}>-\delta$, where virtual states nor longer exist. The relaxation rate of heavier holes, which have smaller energy gaps, is faster than that of electrons, as expected from (11) and (10).

An effective relaxation time, defined as the time when a carrier with initial energy $-\delta$ reaches the state $\varepsilon_{\alpha}=-U / 2$, is found by solving (1) with the right-hand side given by the data plotted in Figure 2 . Thus we obtain $\tau \approx 5$ ps for the hole-hole scattering and $\tau \approx 15$ ps for the electron-electron scattering when the WL carrier density is $n=10^{9} \mathrm{~cm}^{-2}$.

We have demonstrated that resonant scattering considerably enhances relaxation of a single carrier already captured in a QD below a critical level $-\delta$. This initial capturing may be considered a bottleneck of this relaxation process. Few capturing mechanisms can bring the carrier onto those levels, for example, phonon emission by a WL carrier [32]. One may note, however, that the bottleneck appears entirely due to the assumption that the single particle states energy gap remains constant for higher QD levels. This assumption is not applicable for many realistic QD models, where the gap decreases for the upper states, diminishing the bottleneck interval and thus enhancing the capture rate without assistance by phonons. 


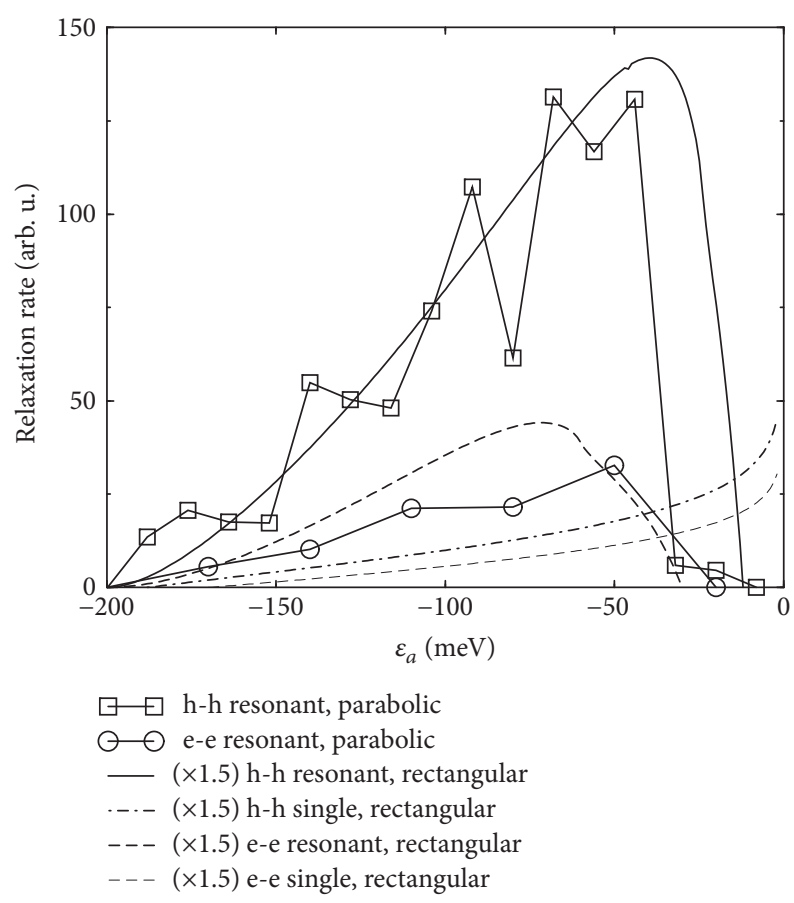

FIGURE 2: Relaxation rate versus energy of a trapped carrier, calculated for parabolic (symbols) and rectangular (continuous lines) dots for electron-electron (e-e) and hole-hole (h-h) scattering.

Moreover, the presence of localized states near the QD makes the capture onto the shallow QD levels more efficient [27].

When the energy of a trapped carrier is close to the ground state the relaxation rate rapidly decreases. The resonance widths become smaller leading to a larger life time of two particle virtual states. However, in this case scattering of WL carriers on a QD with two carriers inside, which is also of a resonant character, ensures a fast relaxation of the two particle trapped states. Similar reasoning can be also applied to relaxation of a general multiexciton complex [12]. Estimation of the corresponding rate may be complicated since the Breit-Wigner formula is likely inapplicable because of the high density of resonances. A detailed discussion of these questions is beyond the aims of this work.

\section{Conflicts of Interest}

The authors declare that there are no conflicts of interest regarding the publication of this paper.

\section{References}

[1] D. Bimberg, M. Grundmann, and N. N. Ledentsov, Quantum Dot Heterostructures, Wiley, New York, NY, USA, 1999.

[2] P. Michler, A. Kiraz, C. Becher et al., "A quantum dot singlephoton turnstile device," Science, vol. 290, no. 5500, pp. 22822285, 2000 .

[3] W. W. Wang, F. M. Guo, and Y. Q. Li, "Modeling and simulation of a resonant-cavity-enhanced InGaAs/GaAs quantum dot photodetector," Advances in Condensed Matter Physics, vol. 2015, Article ID 847510, 6 pages, 2015.
[4] P. Bhattacharya and Z. Mi, "Quantum-dot optoelectronic devices," Proceedings of the IEEE, vol. 95, no. 9, pp. 1723-1740, 2007.

[5] U. Bockelmann and G. Bastard, "Phonon scattering and energy relaxation in two-, one-, and zero-dimensional electron gases," Physical Review B, vol. 42, no. 14, pp. 8947-8951, 1990.

[6] S. Fafard, D. Leonard, J. L. Merz, and P. M. Petroff, "Selective excitation of the photoluminescence and the energy levels of ultrasmall InGaAs/GaAs quantum dots," Applied Physics Letters, vol. 65, no. 11, pp. 1388-1390, 1994.

[7] S. Farfad, R. Leon, D. Leonard, J. L. Merz, and P. M. Petroff, "Phonons and radiative recombination in self-assembled quantum dots," Physical Review B, vol. 52, 1995.

[8] R. Heitz, M. Veit, N. N. Ledentsov et al., "Energy relaxation by multiphonon processes in InAs/GaAs quantum dots," Physical Review B, vol. 56, no. 16, pp. 10435-10445, 1997.

[9] T. S. Sosnowski, T. B. Norris, H. Jiang, J. Singh, K. Kamath, and P. Bhattacharya, "Rapid carrier relaxation in $\mathrm{In}_{0.4} \mathrm{Ga}_{0.6} \mathrm{As} / \mathrm{GaAs}$ quantum dots characterized by differential transmission spectroscopy," Physical Review B, vol. 57, Article ID R9423, 1998.

[10] S. Raymond, K. Hinzer, S. Fafard, and J. L. Merz, "Experimental determination of Auger capture coefficients in self-assembled quantum dots," Physical Review B, vol. 61, no. 24, pp. R16331R16334, 2000.

[11] J. Cruz, F. de Sales, S. da Silva et al., "CW photoluminescence determination of the capture cross-section of self-assembled InAs quantum dots," Physica E: Low-Dimensional Systems and Nanostructures, vol. 17, pp. 107-108, 2003.

[12] H. S. Nguyen, G. Sallen, M. Abbarchi et al., "Photoneutralization and slow capture of carriers in quantum dots probed by resonant excitation spectroscopy," Physical Review B-Condensed Matter and Materials Physics, vol. 87, no. 11, Article ID 115305, 2013.

[13] V. I. Klimov, D. W. McBranch, C. A. Leatherdale, and M. G. Bawendi, "Electron and hole relaxation pathways in semiconductor quantum dots," Physical Review B, vol. 60, article 13740, 1999.

[14] S. Marcinkevicius and R. Leon, "Carrier capture and emission in self-assembled InGaAs/GaAs quantum dots," Physica Scripta T, vol. 79, pp. 79-82, 1999.

[15] Z. Ma, K. Pierz, J. Hübner, and W. W. Rühle, "Nanosecond scale carrier dynamics of self-assembled InAs/AlAs quantum dots studied by time-resolved photoluminescence," Physica E: LowDimensional Systems and Nanostructures, vol. 28, no. 3, pp. 203208, 2005.

[16] Y. Toda, O. Moriwaki, M. Nishioka, and Y. Arakawa, "Efficient carrier relaxation mechanism in InGaAs/GaAs self-assembled quantum dots based on the existence of continuum states," Physical Review Letters, vol. 82, no. 20, pp. 4114-4117, 1999.

[17] R. Ferreira and G. Bastard, "Unbound states in quantum heterostructures," Nanoscale Research Letters, vol. 1, no. 2, pp. 120-136, 2006.

[18] H. S. Ling, C. P. Lee, and M. C. Lo Journal, "Energy-dependent carrier relaxation in self-assembled InAs quantum dots," Journal of Applied Physics, vol. 103, Article ID 124311, 2008.

[19] J. Rihani, N. B. Sedrine, V. Sallet, M. Oueslati, and R. Chtourou, "Observation of many-body Coulomb interaction effects on the photoluminescence spectra of InAs/GaAs quantum dots," Applied Surface Science, vol. 254, no. 10, pp. 3125-3129, 2008.

[20] S. Marcinkevicius, A. Gaardner, and R. Leon, "Rapid carrier relaxation by phonon emission in $\mathrm{In}_{0.6} \mathrm{Ga}_{0.4} \mathrm{As} / \mathrm{GaAs}$ quantum dots," Physical Review B, vol. 64, Article ID 115307, 2001. 
[21] T. S. Shamirzaev, D. S. Abramkin, A. V. Nenashev et al., "Carrier dynamics in InAs/AlAs quantum dots: lack in carrier transfer from wetting layer to quantum dots," Nanotechnology, vol. 21, no. 15, Article ID 155703, 2010.

[22] P. C. Sercel, "Multiphonon-assisted tunneling through deep levels: a rapid energy-relaxation mechanism in nonideal quantumdot heterostructures," Physical Review B, vol. 51, no. 20, pp. 14532-14541, 1995.

[23] X.-Q. Li and Y. Arakawa, "Phonon bottleneck in quantum dots: role of lifetime of the confined optical phonons," Physical Review $B$, vol. 59, p. 5069, 1999.

[24] A. V. Uskov, F. Adler, H. Schweizer, and M. H. Pilkuhn, "Auger carrier relaxation in self-assembled quantum dots by collisions with two-dimensional carriers," Journal of Applied Physics, vol. 81, no. 12, pp. 7895-7899, 1997.

[25] U. Bockelmann and T. Egeler, "Electron relaxation in quantum dots by means of Auger processes," Physical Review B, vol. 46, no. 23, pp. 15574-15577, 1992.

[26] A. L. Efros, V. A. Kharchenko, and M. Rosen, "Breaking the phonon bottleneck in nanometer quantum dots: role of Augerlike processes," Solid State Communications, vol. 93, no. 4, pp. 281-284, 1995.

[27] M. Syperek, M. Baranowski, G. Sęk et al., "Impact of wettinglayer density of states on the carrier relaxation process in low indium content self-assembled (In,Ga)As/GaAs quantum dots," Physical Review B, vol. 87, Article ID 125305, 2013.

[28] K. W. Sun, J. W. Chen, B. C. Lee, C. P. Lee, and A. M. Kechiantz, "Carrier capture and relaxation in InAs quantum dots," Nanotechnology, vol. 16, no. 9, pp. 1530-1535, 2005.

[29] T. Nielsen, P. Gartner, and F. Jahnke, "Many-body theory of carrier capture and relaxation in semiconductor quantum-dot lasers," Physical Review B, vol. 69, Article ID 235314, 2004.

[30] H. H. Nilsson, J.-Z. Zhang, and I. Galbraith, "Homogeneous broadening in quantum dots due to Auger scattering with wetting layer carriers," Physical Review B, vol. 72, Article ID 205331, 2005.

[31] L. D. Landau and E. M. Lifshitz, Course of Theoretical Physics Quantum Mechanics, Oxford Press, 1972.

[32] R. Fereira and G. Bastard, "Phonon-assisted capture and intradot Auger relaxation in quantum dots," Applied Physics Letters, vol. 74, p. 2818, 1999. 

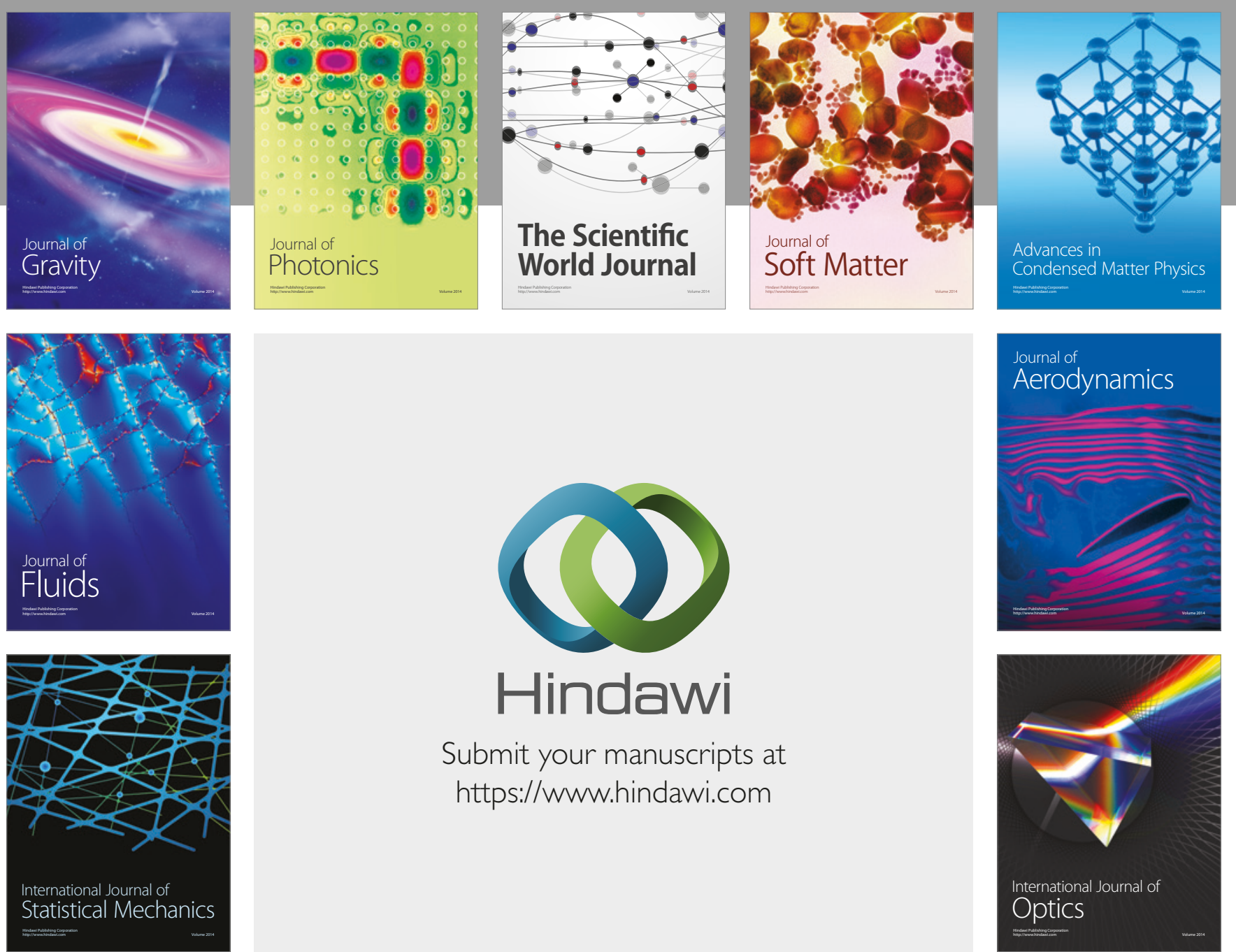

Submit your manuscripts at

https://www.hindawi.com
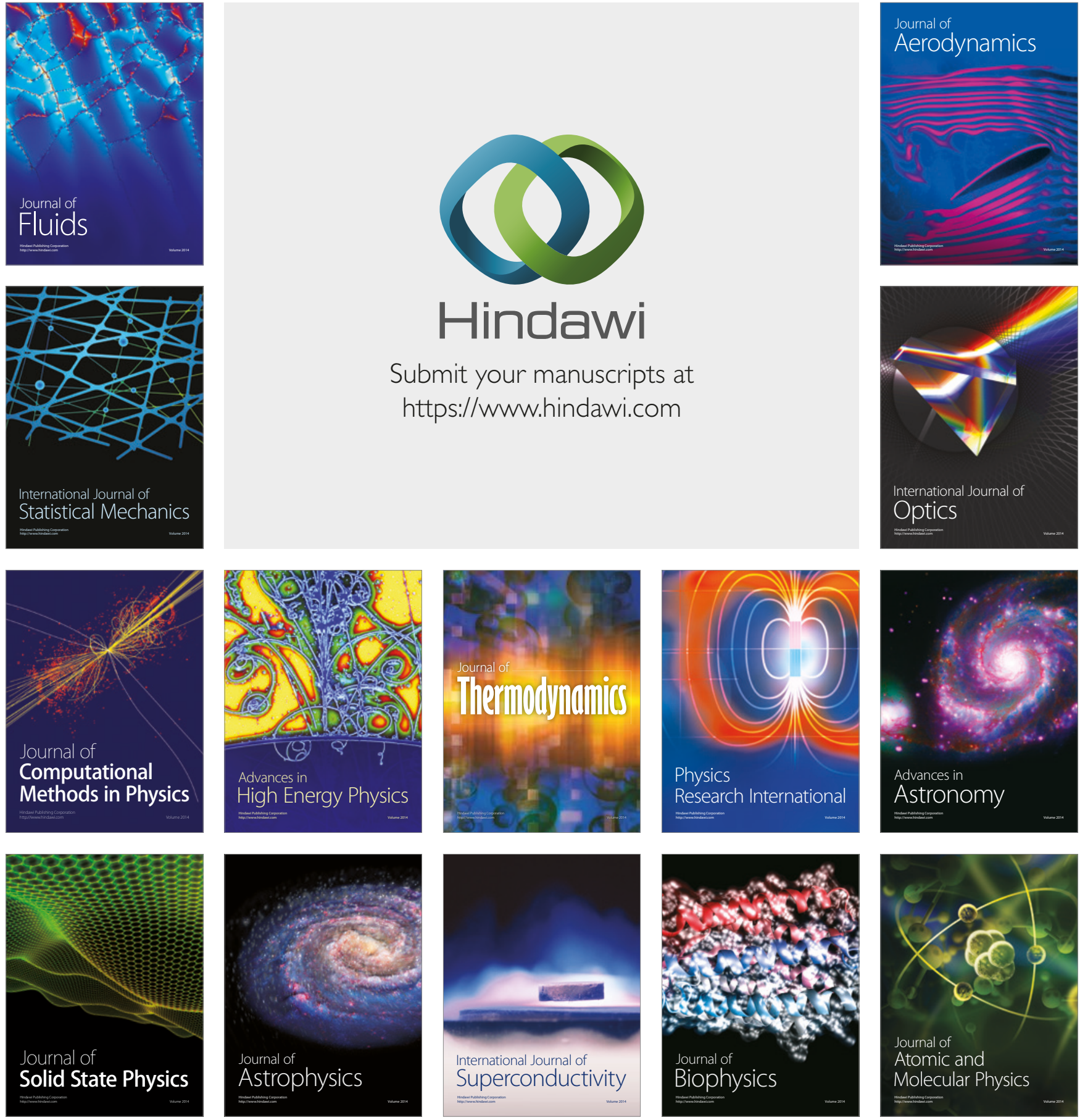